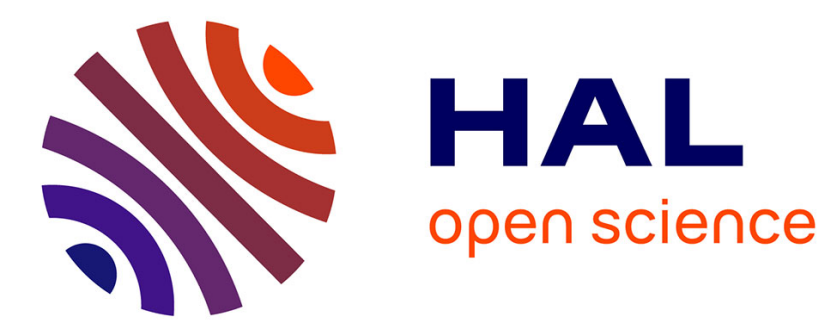

\title{
CCMod: a GATE module for Compton camera imaging simulation
}

\author{
A. Etxebeste, D. Dauvergne, M. Fontana, J.M. Létang, G. Llosá, E. Munoz, \\ J.F. Oliver, É Testa, D. Sarrut
}

\section{To cite this version:}

A. Etxebeste, D. Dauvergne, M. Fontana, J.M. Létang, G. Llosá, et al.. CCMod: a GATE module for Compton camera imaging simulation. Physics in Medicine and Biology, 2020, 65 (5), pp.055004. 10.1088/1361-6560/ab6529 . hal-02497878

\section{HAL Id: hal-02497878 https://hal.science/hal-02497878}

Submitted on 22 Dec 2020

HAL is a multi-disciplinary open access archive for the deposit and dissemination of scientific research documents, whether they are published or not. The documents may come from teaching and research institutions in France or abroad, or from public or private research centers.
L'archive ouverte pluridisciplinaire HAL, est destinée au dépôt et à la diffusion de documents scientifiques de niveau recherche, publiés ou non, émanant des établissements d'enseignement et de recherche français ou étrangers, des laboratoires publics ou privés. 


\title{
CCMod: a GATE module for Compton Camera imaging simulation
}

\author{
A. Etxebeste ${ }^{1}$, D. Dauvergne ${ }^{2}$, M. Fontana ${ }^{3}$, J. M. Létang ${ }^{1}$, \\ G. Llosá ${ }^{4}$, E. Munoz ${ }^{4}$, J. F. Oliver ${ }^{4}$, É. Testa ${ }^{3}$ and \\ D. Sarrut ${ }^{1}$ \\ ${ }^{1}$ Université de Lyon, CREATIS, CNRS UMR5220, Inserm U1044, INSA-Lyon, \\ Université Lyon 1, France. \\ ${ }^{2}$ LPSC, Université Grenoble-Alpes, CNRS/IN2P3 UMR 5821, Grenoble, France. \\ ${ }^{3}$ Université de Lyon, CNRS/IN2P3, UMR 5822, IPNL, F-69622 Villeurbanne, \\ France. \\ ${ }^{4}$ Instituto de Física Corpuscular, CSIC/Universitat de València, Valencia, Spain. \\ E-mail: ane.etxebeste@creatis.insa-lyon.fr
}

\begin{abstract}
.
Compton cameras are gamma-ray imaging systems which have been proposed for a wide variety of applications such as medical imaging, nuclear decommissioning or homeland security. In the design and optimization of such a system Monte Carlo simulations play an essential role. In this work, we propose a generic module to perform Monte Carlo simulations and analyses of Compton Camera imaging which is included in the open-source GATE/Geant4 platform. Several digitization stages have been implemented within the module to mimic the performance of the most commonly employed detectors (e.g. monolithic blocks, pixelated scintillator crystals, strip detectors...). Time coincidence sorter and sequence coincidence reconstruction are also available in order to aim at providing modules to facilitate the comparison and reproduction of the data taken with different prototypes. All processing steps may be performed during the simulation (on-the-fly mode) or as a post-process of the output files (offline mode). The predictions of the module have been compared with experimental data in terms of energy spectra, angular resolution, efficiency and back-projection image reconstruction. Consistent results within a 3-sigma interval were obtained for the energy spectra except for low energies where small differences arise. The angular resolution measure for incident photons of $1275 \mathrm{keV}$ was also in good agreement between both data sets with a value close to $13^{\circ}$. Moreover, with the aim of demonstrating the versatility of such a tool the performance of two different Compton camera designs was evaluated and compared.
\end{abstract}

Keywords: Monte Carlo, simulation, gamma imaging, Compton camera

\section{Introduction}

Compton Cameras (CCs) are actively collimated gamma-ray imaging devices which have been originally designed for astronomy applications. Their first application in medical imaging was proposed in (Todd et al. 1974). Thenceforth, there has been an interest in their application in the field of nuclear medical imaging (Han et al. 2008, Seo et al. 2008, Harkness et al. 2009, Karimian et al. 2009, Alnaaimi et al. 2011, Odaka et al. 2012, Calderón et al. 2014, Fontana et al. 2017). In addition, there is also a growing interest in their use to image the prompt gamma 
emission produced during proton therapy for in-vivo range assessment (Richard et al. 2011, Peterson et al. 2010, Kormoll et al. 2011, Polf et al. 2015, Hilaire et al. 2016, Solevi et al. 2016, Rohling et al. 2017).

In nuclear medicine, Single Photon Emission Computed Tomography (SPECT) Anger gamma-camera is nowadays one of the most widespread imaging techniques for diagnosis. The main drawback of this technique is that the sensitivity is inversely proportional to the spatial resolution due to the use of mechanical collimation. In order to overcome this limitation, the use of active collimation systems such as CCs has been proposed. They usually consist of several position and energy sensitive gamma ray detectors arranged in scatterer and absorber planes working in time coincidence. Using the kinematics of Compton scattering, the origin of the gamma-ray can be constrained to a cone surface. As a result of the intersection of different cones, information of the activity distribution can be obtained.

However, to our knowledge, commercial devices have only been proposed for astronomy or for envirommental measurements in homeland security but not for medical applications, even if several prototypes have been built (Llosá et al. 2006, Seo et al. 2010, Alnaaimi et al. 2011, Takeda et al. 2012, Kurosawa et al. 2012, Kishimoto et al. 2015, McCleskey et al. 2015, Thirolf et al. 2016, Golnik et al. 2016, Muñoz et al. 2017, Lee et al. 2017). The high performance required in terms of energy, spatial and time resolution when CCs are employed in medical applications together with the complexity of the image reconstruction algorithms have prevented them from becoming a commercial medical imaging system. These gamma-ray imaging devices need to be further investigated and optimized to reach the clinical standards for which accurate Monte Carlo (MC) simulations are required.

MC simulations play an essential role in the design and optimization of these systems (Richard et al. 2011), the validation of reconstruction algorithms (Andreyev et al. 2016) and the extrapolation of the behaviour of these devices to unavailable situations in order to predict their potential (Muñoz et al. 2017). Several particle-tracking MC simulation software packages have been used to simulate CCs, such as CIS (Lee et al. 2009), MEGAlib (Zoglauer et al. 2006), GAMOS (Arce et al. 2008, Harkness et al. 2012), Geant4 (Richard et al. 2011, Peterson et al. 2010, Rohling et al. 2017, Fontana et al. 2017), SWORD (Novikova et al. 2006) or BEAMnrc/EGSnrc (Sinclair et al. 2009). GATE/Geant4 (Jan et al. 2011, Sarrut et al. 2014) is an open-source and collaborative simulation platform dedicated to medical physics applications which is based on the well established general purpose Geant4 software (Allison et al. 2016). Nowadays, GATE is commonly used for emission tomography simulations (Gillam and Rafecas 2016), notably due to the broad range of experimental settings that can be simulated by using macro language. GATE already contains dedicated modules for SPECT and PET imaging.

In this work, we present an extension of GATE toolkit with the aim of supporting CC simulations. This new Compton Camera Module (CCMod) facilitates the investigation of $\mathrm{CC}$ systems and realistic comparisons between different prototypes in an environment that allows to simulate several imaging devices, radiotherapy treatments and perform dosimetry calculation in the same framework. As an example, simulations of protontherapy beam lines (Grevillot et al. 2011, Grevillot et al. 2012) are already available and may be used to generate realistic distributions of promptgammas (Gueth et al. 2013, Huisman et al. 2016) that may be investigated with CCs. Moreover, GATE already contains specific modules for medical physics simulations, which allow to easily insert a voxelized CT image from a DICOM file or to read and 
analyze list mode format among others. In this work, the predictions of CCMod have been compared with experimental data taken with MACACO prototype built at IFIC-Valencia (Muñoz et al. 2017). The developed tools have been employed to illustrate their capability to characterize and enhance experimental data by developing dedicated filtering criteria to reduce the fraction of background events. Furthermore, the performance of two different CCs has been characterized in terms of efficiency and angular resolution showing the versatility of this open-source module.

\section{Materials and Methods}

All the simulation studies included in this work were performed with Geant4 version 10.5 and GATE version 8.2.

\subsection{Compton Camera module}

2.1.1. Compton Camera principles. CCs employ electronic collimation based on Compton kinematics. A conventional CC is comprised of a scatterer and an absorber detector layer working in time coincidence. Ideally, the gamma-ray undergoes a Compton interaction in the closest detector layer, referred to as scatterer, where the recoil electron deposits its energy, and the scattered photon is absorbed in the absorber. Using the kinematics of Compton scattering the incident trajectory of the gamma-ray can be constrained to a cone surface. However, in experimental data there is a non-negligible percentage of invalid events that contribute to the background such as partial energy absorption events (recoil electron or scattered photon escape), incorrectly ordered interactions and randoms.

CCs typically consist of low-Z material for the scatterer and high-Z material for the absorber in order to maximize the probability of Compton scattering in the scatterer and photoelectric absorption of the scattered photon in the absorber. The energy resolution of the scatterer is particularly important to accurately determine the scattering angle. To this end, a low $\mathrm{Z}$ material is also of special interest to reduce the effect of Doppler energy broadening, Compton profile, due to the initial momentum of the bound electron (Ordonez et al. 1997).

In most proposed designs, the device is comprised of a scintillator detector for the absorber and one or several detector layers for the scatterer based on different technologies such as scintillator crystals coupled to compact SiPM/MPPC arrays (Muñoz et al. 2017, Kishimoto et al. 2015), semiconductor detectors (Seo et al. 2010, Thirolf et al. 2016, Golnik et al. 2016, Lee et al. 2017, Fontana et al. 2017) or gaseous detectors (Kurosawa et al. 2012). In addition, CCs composed only of semiconductor detector layers (Alnaaimi et al. 2011, Takeda et al. 2012, McCleskey et al. 2015) have been successfully built and tested.

The aim of CCMod is to provide a framework where different system configurations can be easily simulated in a medical environment. It was designed to model the most common CC configuration with a scatterer and an absorber. However, it can be adapted to accommodate other designs such as one single position sensitive detector system that acts as scatterer and absorber at the same time (Lehner et al. 2004, Mihailescu et al. 2007, Maier et al. 2017, Montémont et al. 2017) that can be of interest in homeland security applications. 
2.1.2. GATE module. CCMod has been implemented as a dedicated GATE actor that follows the same structure to store and process the interaction of particles as the SPECT and PET scanner systems (Jan et al. 2011). Portions of the geometry are designated as Geant4's sensitive detectors where interactions are recorded. Here, the absorber and scatterer layers store information about all particle interactions from now on referred to as hits. A hit contains the interaction process type (Compton, photoelectric, ionization...), the layer name, the position interaction, the deposited energy, the time and information on parent particle among others.

Individual detector layer responses obtained from the aggregation of the hits are referred to as singles. The singles are then sorted in time and associated in coincidences. Finally, the singles within each coincidence are ordered generating a sequence coincidence. This sequence determines the cone surface. This processing from interactions to cone information is divided into three different steps namely digitizer, sorter and coincidence sequence reconstruction which are explained in detail in sections 2.1.3 to 2.1.5. At each step the corresponding output data (hit collection, singles, coincidence, sequence coincidence) are available to the user. All processing steps may be performed during the simulation (on-the-fly mode) or on the simulation output files (offline mode).

A similar data format for hit collection and singles as for SPECT and PET modules has been used to be able to share the digitizer modules and sorter between the different imaging devices. Hence, some of the already available modules for SPECT and PET systems (Jan et al. 2004), e.g. energy resolution, time resolution, energy window, spatial resolution, and dead-time, can be directly applied in CCMod. Several new modules, briefly described in section 2.1.3, have been developed to meet the needs of some of the most commonly employed CC configurations. The features included in these additional modules, that can be equally applied to SPECT/PET systems, can be of interest to simulate more accurately and further understand the acquired data also in those imaging modalities.

2.1.3. Digitizer. The digitizer consists of applying sequentially a chain of modules to the hit collection to mimic the individual detector response creating the singles. In order to reproduce accurately the response of a detector, the modules that compose the digitizer chain must be carefully selected.

With the aim of modeling the response of the most commonly employed detectors in CCs such as semiconductor, pixelated and monolithic scintillator detectors (see 2.1.1), several modules have been implemented. These modules can be classified into three different categories. The first one corresponds to those that spatially regroup hits to take into account the characteristics of the signal generation in the detector such as grid discretization module which simulates the response of pixelated or strip detectors. The second one corresponds to modules that modify the observable values of the singles such as Depth of Interaction (DoI) modeling or 3-dimensional spatial resolution module. The last category comprises those modules that filter the singles such as energy threshold modules. Figure 1 shows the general structure of the digitization chain divided into the aforementioned three categories applied to a 5-hit-collection composed of three photon interactions, two Compton scattering and a photoelectric absorption, and two secondary electronic interactions. As an example, the response of a monolithic block readout by position sensitive photo-detectors is approached by applying a hit regrouping module to collect the total energy deposited by the hits and provide the energy weighted centroid position. 
Since in CCs different detector layers have usually different roles and characteristics, these modules have been designed so that they can be applied locally to a specified sensitive volume. In addition, with the aim of recovering Compton kinematics, a module that provides the information of the incident photon before and after each interaction has been developed. Additional modules can be easily added by the users to take into account unforeseen situations in specific configurations.

The digitization of optical photons has not been considered in CCMod. The performed simulations do not include the generation and transport of optical photons which highly increase the computational cost (Bonifacio et al. 2010). However, several digitization modules, such as DoI module which models the influence of the light distribution on the 3-dimensional spatial resolution of the detector, have been implemented aiming at reproducing the response of scintillator crystals.

2.1.4. Sorter. In Geant4, events are created sequentially. However, singles are not necessarily arriving to the sorter in chronological order. Therefore, they are ordered by their simulation time value before being sorted in time, generating coincidences when they are detected within the same Time Coincidence Window (TCW). To this end, the sorter developed in GATE for PET systems (Strydhorst and Buvat 2016) has been adapted for CCMod. By default, Single Window method is selected where as long as a coincidence window is opened, no other single can open its own coincidence window. Multiple Window method is also available as for PET systems. An additional option has been included to allow only singles in the absorber layer to open its own window (absorber coincidence trigger). The criterion set for CC coincidence processing acceptance is that at least two of the singles within the TCW are recorded in different detector layers.

2.1.5. Coincidence Sequence Reconstruction (CSR). The CSR is a coincidence processor which modifies the order of the singles within a coincidence to generate a sequence coincidence. In CCs, CSR plays an essential role since the correct sequence of singles is necessary to determine the correct cone surface where the source is located. By default, the singles within a coincidence are ordered according to their detection time, i.e by increasing time-stamp value. This policy is referred to as ideal. However, since the time resolution of the detector layers is generally not good enough to identify the correct interaction sequence, different policies are adopted. CCMod includes several policies namely random, distance to the source and the above described ideal policy. The distance to the source policy orders the singles by increasing distance to the source in the perpendicular direction to the camera, i.e. first singles in the first layer. Furthermore, a policy based on the so-called revan analyzer from MEGAlib (Zoglauer et al. 2008), known as Classic Coincidence Sequence Reconstruction (CCSR) has been included.

Coincidence processing modules already available for PET systems, such as deadtime or memory buffer can also be employed in the CCMod.

\subsection{Comparison with experimental data}

2.2.1. MACACO prototype The predictions of CCMod were compared with experimental data taken at IFIC-Valencia with the first version of MACACO (Medical Applications CompAct COmpton camera) prototype (Muñoz et al. 2017). The prototype is based on $\mathrm{LaBr}_{3}$ monolithic crystals coupled to SiPMs. The system 


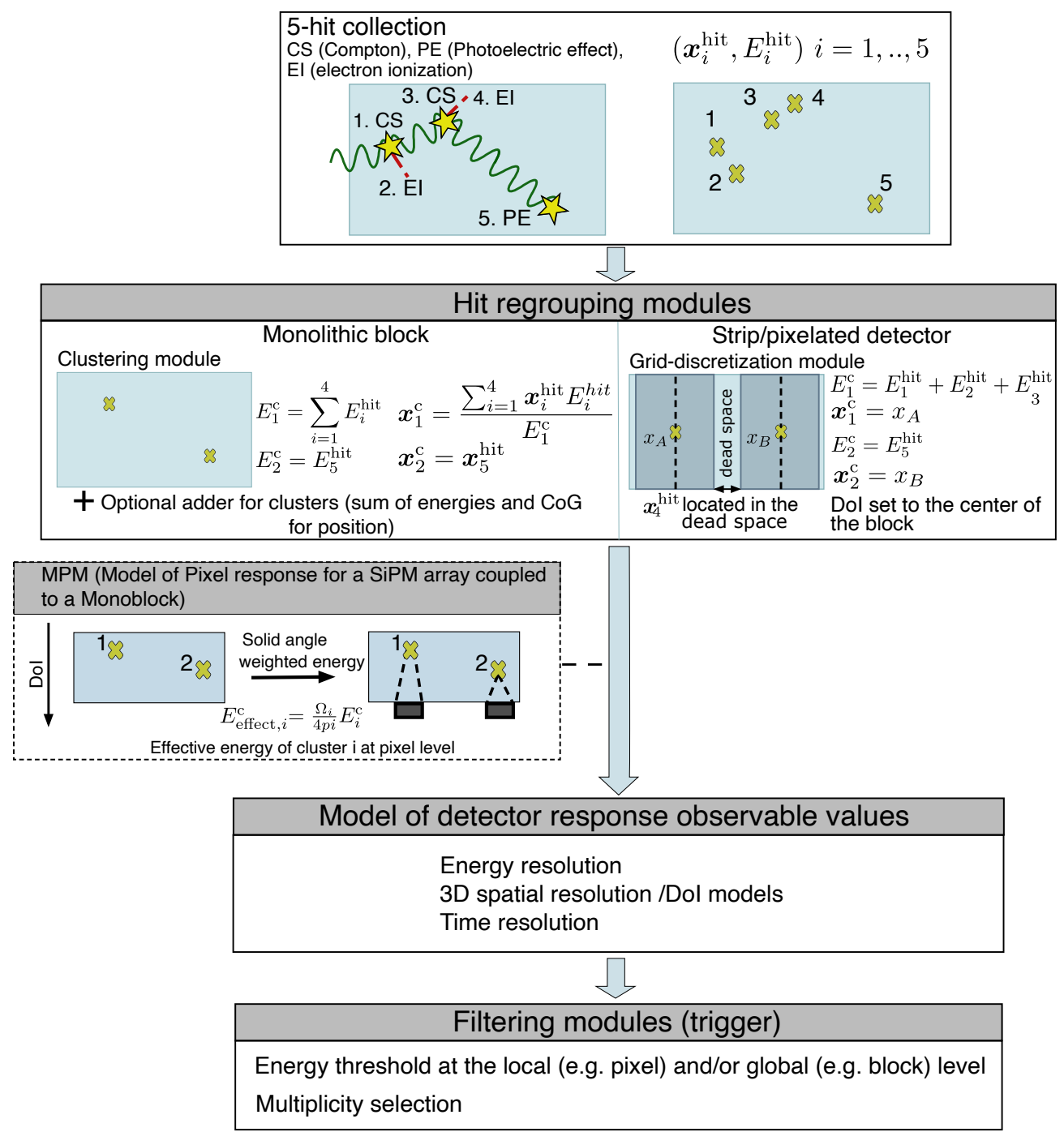

Figure 1: Scheme of the digitization chain applied to a 5 -hit-collection where $\boldsymbol{x}_{i}^{\text {hit }}$ and $E_{i}^{\text {hit }}$ are $i$-th hit position and energy deposition respectively. For simplicity, the set of possible hits associated to the recoil electron are represented by a single hit. The position and the energy deposition of clusters after applying a hit regrouping module are represented by $\boldsymbol{x}_{i}^{\mathrm{c}}$ and $E_{i}^{\mathrm{c}}$ respectively.

setup, the so-called configuration 1 in (Muñoz et al. 2017), was composed of two layers separated by $50 \mathrm{~mm}$. Both layers were made of monolithic scintillator crystals coupled to SiPM arrays with active area elements of $3 \times 3 \mathrm{~mm}^{2}$. The crystal sizes were $27.2 \times 26.8 \times 5 \mathrm{~mm}^{3}$ and $32.0 \times 36 \times 10 \mathrm{~mm}^{3}$ for the first and second layer respectively. The first layer was based on four Hamamatsu MPPC S11830-3340MF monolithic arrays whereas the second one was based on an older version S11064-050P(X1) with larger gaps between the pixels. More details can be found in (Muñoz et al. 2017). A second version of the prototype is currently under development based on higher 
performance SiPM arrays with the aim of improving the energy resolution of the prototype. The SiPM, model S13361-3050AE-08, is based on TSV (Through Silicon Via) technology. Further information is available in (Barrio et al. 2018).

The reported measurements, unless otherwise stated, were taken with the first version of the prototype and a ${ }^{22} \mathrm{Na}$ source of $847 \mathrm{kBq}$ activity located in the center of the first plane at a perpendicular distance of $53 \mathrm{~mm}$ (see figure 2). The acquisition discriminator threshold applied at pixel level was set about $70 \mathrm{keV}$ for each plane (sum of the values in all 64 pixels) (Muñoz et al. 2017).

\subsubsection{Simulation characteristics}

Geometry Two sets of simulations were performed to reproduce the experimental data: a simplified geometry in which only the volumes corresponding to the sensitive detector layers (scintillator crystals) were included and another one in which additional insensitive components of the prototype i.e. crystal encapsulation, crystal holder, PCBs (printed circuit boards) and the SiPMs, were included in order to take into account their contribution to photon scattering. The compositions, densities and thicknesses of these materials are not exactly known and they were not optimized. The camera was simulated in air. Both geometries are shown in figure 2.

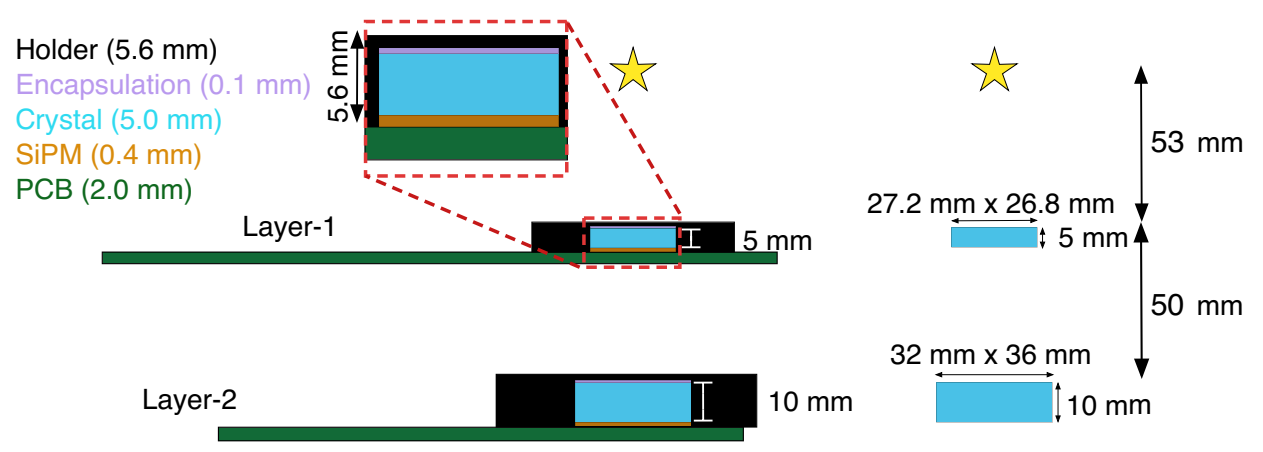

Figure 2: Simulated geometry. Left, geometry with the main parts of the passive materials of the experimental set up included. Right, simplified geometry.

Processing The first step of the processing was the digitization of the hits to reproduce the response of the detector-layers, see section 2.1.3. The digitization chain was designed with the aim of modeling the experimental response of a monolithic crystal coupled to a SiPM matrix with an acquisition discriminator threshold applied at pixel level.

Following the general structure shown in figure 1, a hit regrouping module was firstly applied. The clustering module was selected to group the hits within an acceptance distance of $3 \mathrm{~mm}$, which corresponds to the SiPM pixel size. Then, the maximum deposited energy in a $\mathrm{SiPM}$ pixel of the array $\left(E_{\text {effect }, i}^{c}\right)$ was approximated analytically without the generation and transport of optical photons using the MPM, namely the Model of Pixel response for a SiPM array coupled to a Monoblock, see figure 1. This effective energy at pixel level was obtained for each cluster by weighting 
its energy by the fraction of the solid angle subtended by a virtual pixel centered at the cluster X-Y position at the readout surface of the crystal. Then, the conventional adder was applied to generate a single signal per layer and per event where the energy corresponds to the total deposited energy of all the clusters in the layer and the position to the energy weighted centroid position. Pile up effect was not included since it was negligible in the experimental data.

After the hit regrouping modules, energy and spatial resolution modules were applied. The energy resolution was set to $8.5 \%$ at $511 \mathrm{keV}$ in the first layer, with an energy dependence following the inverse square root law policy. In the second layer however a slightly worse resolution of $12.5 \%$ at $511 \mathrm{keV}$ was set to match the experimental data. The influence of the temperature variations on the energy resolution was not modeled. Regarding the transverse spatial resolution, a $2 \mathrm{~mm}$ Gaussian blurring was applied. In this work, the DoI uncertainty was modelled as an exponential function with a coefficient of $-0.3507 \mathrm{~mm}^{-1}$ (Cabello et al. 2015) and a value of $8 \mathrm{~mm}$ FWHM at the entrance of the crystal. However, different models were tested for DoI, such as dual layer model or Gaussian blurring for the DoI uncertainty, yielding equivalent results.

Finally, a local threshold was applied at pixel level where if at least the effective energy of one of the clusters $\left(E_{\text {effect }, i}^{c}\right)$ was above the threshold the whole event was accepted for processing. Due to the uncertainties in the applied experimental discriminator threshold which was set to get a global energy threshold in each layer of above $\sim 70 \mathrm{keV}$ (Muñoz et al. 2017), and the simplified model employed to reproduce it, discrepancies between the simulated and experimental accepted singles distribution are expected. In order to minimize them, a slightly higher global energy threshold of $85 \mathrm{keV}$ was also applied to the total energy deposition in each layer to both data sets.

After the digitization process, the generated singles were sorted using a TCW of $50 \mathrm{~ns}$. The singles within the sequence coincidence were ordered by increasing axial distance to the source, i.e. first singles in the first layer. It was considered that the majority of coincidences corresponds to forward scattering in the scatterer followed by an interaction in the second layer.

Source ${ }^{22} \mathrm{Na}$ source was simulated with an activity of $847 \mathrm{kBq}$ and a diameter of $0.5 \mathrm{~mm}$ that correspond to the experimental values. The source was embedded in a plastic phantom that represents the source encapsulation and facilitates the positron annihilation.

The intrinsic activity of ${ }^{138} \mathrm{La}$ of the $\mathrm{LaBr}_{3}$ crystal and naturally occurring radioactive materials were not included since the detected count rates due to the natural radioactivity were negligible $(<1 \%)$ compared to those generated by the employed external source. Natural radioactivity can be included in the simulation with an additional source.

Physics list and cuts The option 4 of the standard electromagnetic physics list, which includes Doppler broadening effect, was selected. The employed model for Compton scattering for energies below $20 \mathrm{MeV}$ was LowEPComptonModel (Brown et al. 2014) with G4EMLOW7.7 database. Atomic de-exitation processes such as fluorescence, Auger and PIXE (Particle-Induced X-ray Emission) effects were activated for all volumes (sensitive and insensitive materials). Cuts values were set to $0.1 \mathrm{~mm}$ which corresponds to one order of magnitude less than the smallest dimension of the sensitive 
detectors.

Time A simulation time of $200 \mathrm{~s}$ was set leading to around 20000 accepted coincidences. The simulation lasted around 14 hours on a single Intel(R) Xeon(R) CPU E5-2620@2.00GHz.

2.2.3. Figures of Merit The verification was made through the comparison of different figures of merit namely energy spectra of singles, energy spectra of coincidences, scattering angle, angular resolution and efficiency. In addition, reconstructed images obtained from the back-projection onto the plane of the source were compared.

Scattering angle The estimated scattering angles obtained from the Compton kinematics $\theta_{C}$ were compared to the ones obtained from geometrical method $\theta_{G}$ following eq. (1a) and eq. (1b) respectively:

$$
\begin{aligned}
& \cos \left(\theta_{C}\right)=1-\frac{m_{e} c^{2} E_{1}}{E_{0}\left(E_{0}-E_{1}\right)} \\
& \cos \left(\theta_{G}\right)=\frac{\vec{g}_{10} \cdot \vec{g}_{21}}{\left|\vec{g}_{10}\right|\left|\vec{g}_{21}\right|}
\end{aligned}
$$

where $E_{1}$ is the energy deposited in the Compton scattering, $E_{0}$ is the incident energy, $m_{e}$ is the rest mass energy of the electron, $\vec{g}_{10}$ is the vector that goes from the source position to the first interaction position and $\vec{g}_{21}$ from the first interaction to the second interaction position. Due to the error associated with the manual positioning of the source in the experimental set-up, the position of the source was corrected taking into account the reconstructed image.

For the comparison with experimental data, the initial energy $E_{0}$ was estimated from the sum of the energy deposition in the layers. When the total deposited energy was below $600 \mathrm{keV}, E_{0}$ was assigned to the $511 \mathrm{keV}$ photon and otherwise to the $1275 \mathrm{keV}$ photon.

Angular Resolution The error distribution of the scatter angles was computed as the difference between $\theta_{G}$ and $\theta_{C}$ (Zoglauer and Kanbach 2003, Seo et al. 2011, Odaka et al. 2012, Mizumoto et al. 2015). The Angular Resolution Measure (ARM) value is given by the FWHM of the distribution. Its magnitude is related to the resolution of the final image. Therefore, it enables to study the resolution excluding the dependence of the reconstruction methods.

In order to fit the non-Gaussian distribution of the ARM, different functions have been employed in the literature: a Voigt function (a convolution of the Gaussian and Lorentzian distributions) (Seo et al. 2011, Odaka et al. 2012), a Lorentzian function (Mizumoto et al. 2015) or a superposition of different Lorentzian functions (Zoglauer and Kanbach 2003). In this work, the ARM value was obtained by fitting the distribution with a Lorentzian function.

Relative detection efficiency The relative detection efficiency was calculated as the fraction of events emitting a photon within the solid angle subtended by the first 
detector layer that produces a detected coincidence. In order to compare the obtained value with the experimental value reported in (Muñoz et al. 2017) for the $1275 \mathrm{keV}$ photons of the ${ }^{22} \mathrm{Na}$ source, the coincidences with a total deposited energy comprised between 600 and $1350 \mathrm{keV}$ were selected.

\subsection{CCMod as a facilitator towards more accurate results}

CCMod can be employed to better understand the experimental data and to get more accurate results. The ground-truth provided by the developed tool on a validated simulation can be employed to define ad-hoc filters based on observable values to improve experimental data by reducing the fraction of invalid events.

In this section, the developed simulation was employed to illustrate the capability of the CCMod to characterize the signature of the deposited energy of random coincidences. This type of invalid events was chosen for illustration purposes. The studied experimental data were taken with a ${ }^{22} \mathrm{Na}$ source which leads to several photons per decay ( $511 \mathrm{keV}$ and $1275 \mathrm{keV}$ ). Therefore, the information of the primary photon that produces each interaction, included in the CCMod, was employed to distinguish between random coincidences generated by the detection of different photons coming from the same decay process (same event identification) and photons coming from two different decays (different event identification).

In addition, the angular error distribution was studied to evaluate the impact of the energy threshold applied experimentally and to characterize the energy signature of the events with large angular error value with the aim of reducing the fraction of background events.

\subsection{Comparison of the performance of different prototypes}

The comparison of the performances between MACACO and CLaRyS prototypes was performed. The CLaRyS collaboration includes four institutions: Institut de Physique Nucleaire de Lyon (IPNL), Centre de Recherche en Acquisition et Traitement de l'Image pour la Santé de Lyon (CREATIS), Centre de Physique de Particules de Marseille (CPPM) and Laboratoire de Physique Subatomique et Cosmologie de Grenoble (LPSC). The prototype under development by the CLaRyS collaboration is described below.

2.4.1. The CLaRyS Compton Camera prototype The CLaRyS prototype was originally designed and optimized for hadrontherapy purposes. However, the simulated geometry of the absorber corresponds to the one presented in (Fontana et al. 2017), which was adapted to be as close as possible to a commercial SPECT-Anger camera device (GE Healthcare Infinia system). The scatterer detector is composed of seven parallel double sided silicon strip detectors (DSSDs) separated by $10 \mathrm{~mm}$ distance from each other. The absorber, $210 \times 280 \times 30 \mathrm{~mm}^{3}$ in size, is composed of $6 \times 8 \mathrm{BGO}$ streaked blocks. Each block was simulated as an $8 \times 8$ pixel matrix. The distance between the last silicon plane (center) and the center of the absorber is $150 \mathrm{~mm}$. The DSSDs are $90 \times 90 \times 2 \mathrm{~mm}^{3}$ in size with $2 \times 64$ strips in a $1.4 \mathrm{~mm}$ pitch. The camera was simulated in air.

Processing. Different digitization chains were applied to model the responses of scatterer and absorber layers following the specifications described in (Fontana 
et al. 2017). Regarding the scatterer, a grid discretization module was applied to model the charge collection of the DSSDs where 64 strips of $1.3 \mathrm{~mm}$ active size in a $1.4 \mathrm{~mm}$ pitch were simulated in each transverse direction. An activation threshold of $50 \mathrm{keV}$ was set at strip intersection level. An energy resolution of $2.5 \%$ at $200 \mathrm{keV}$ with an energy dependence following the inverse square root law policy was additionally applied. Regarding the absorber layer, the scintillator crystal was simulated with $6 \times 8$ blocks made of $8 \times 8$ pixels each with an active area of $4.375 \mathrm{~mm}^{2}$ in size. The readout was performed at block level. The signal of each block corresponded to the total energy deposition taking into account the 64 pixels, and the position was given by the energy weighted centroid position. An activation threshold of $100 \mathrm{keV}$ was set at the block level. An energy resolution of $21 \%$ at $662 \mathrm{keV}$ with an energy dependence following the inverse square root law policy was set to match the experimental performance. In both cases, the transverse spatial resolution was determined by the applied discretization module and the DoI was set to the center of the detector layer. After the digitization process, the singles were sorted in time using the same TCW value as in MACACO prototype (50 ns). For the CSR, forward scattering policy was also selected.

Multiple coincidences may contain useful information about the source in CLaRyS prototype since it is composed of several scatterer layers and a pixelated absorber. However, for comparison purposes with the MACACO configuration composed of two monolithic crystals, only coincidences composed of one single in each detector type were considered.

The physics list and cuts described in 2.2 were applied.

2.4.2. Evaluation The performance of both prototypes was studied in terms of detection efficiency normalized to the geometrical acceptance of the scatterer (relative detection efficiency) and ARM. The relative detection efficiency for CLaRyS prototype was studied for coincidences composed of two singles. To this end, monochromatic point-like gamma sources related to radioemitters already suggested for nuclear medicine applications (Fontana et al. 2017) emitting in a range comprised between $245-2614 \mathrm{keV}$ were employed. The source was located $53 \mathrm{~mm}$ far from the surface of the first layer as for MACACO experimental data. In order to make a fair comparison the passive structure of MACACO prototype was not included in this study. An acquisition time of $200 \mathrm{~s}$ and an activity of $847 \mathrm{kBq}$ were simulated. The computational time for CLaRyS prototype was between 3 and 6 hours depending on the incident energy whereas for MACACO prototype was around 2 hours on a single Intel(R) Xeon(R) CPU E5-2620 @ 2.00GHz.

\section{Results}

\subsection{Comparison with MACACO experimental data}

3.1.1. Energy spectra of singles In order to evaluate if the structure of the prototype plays a significant role in the measurements, the energy spectrum of the singles was studied. The comparison of the spectra between experimental data and the two sets of simulations, the simplified one and the one that includes part of the passive structure of the prototype, without applying any energy threshold is shown in figure $3(\mathrm{a})$ and figure 3(b) for the first and the second layer respectively.

In the singles spectra we can observe the photo-peaks, at $511 \mathrm{keV}$ and $1275 \mathrm{keV}$, together with the Compton edges in each detector layer. In addition, the absorption 


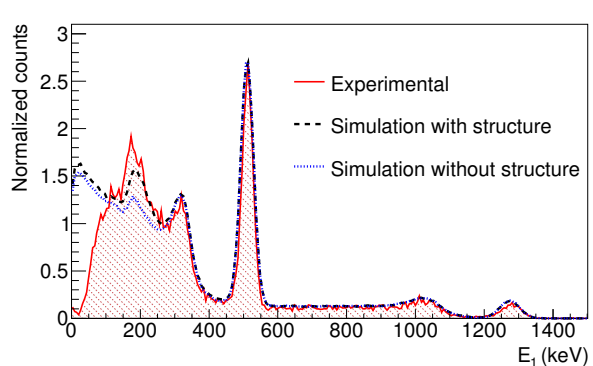

(a)

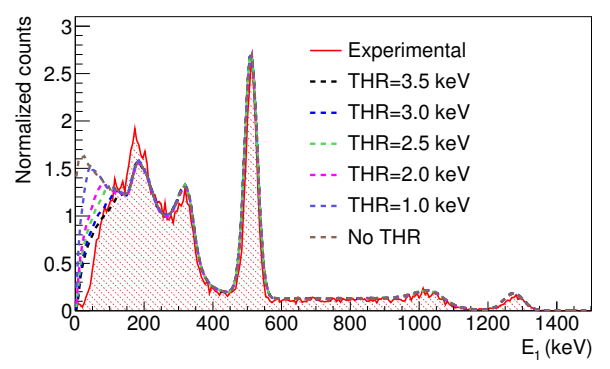

(c)

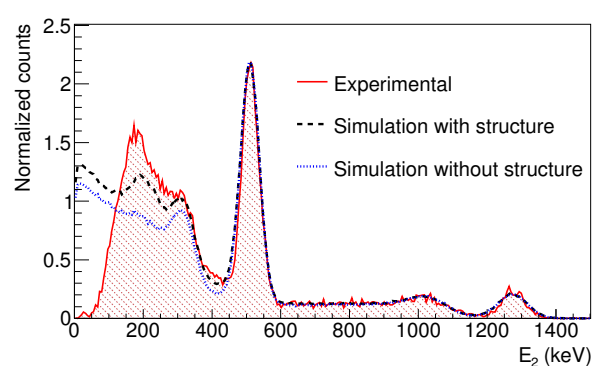

(b)

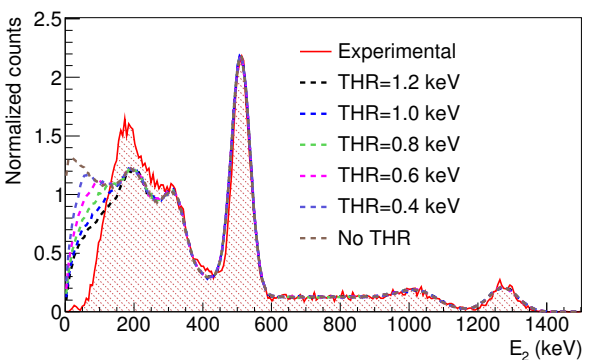

(d)

Figure 3: Simulated and experimental singles energy spectra with and without including the passive structure in (a) layer 1 and (b) layer 2. Comparison between experimental and simulated energy spectra when the passive material is included and different values for the local threshold are applied in (c) layer 1 and (d) layer 2.

of the photons undergoing back-scattering outside the studied detector layer, with energies around $\sim 170 \mathrm{keV}$ and $\sim 213 \mathrm{keV}$ respectively, can be clearly seen. The energy spectrum of both simulation types above the first photo-peak was equivalent. However, when the structure of the prototype was considered, there was an increment of detected singles at low energies resulting in a better agreement with experimental data. In both detector layers, the recovered intensity of the back-scattering fronts was higher and better matching the experimental data. Moreover, for the second detector layer, the Compton edge was also better recovered. Hence, all of the following simulations include the passive material.

Another difference that can be found in figure 3(a) and figure 3(b) was the lack of events depositing an energy below $\sim 100 \mathrm{keV}$ in experimental data with respect to the simulations. This effect is due to the discriminator threshold applied at individual SiPM pixel level for the experimental acquisition, which does not result in a unique global threshold value at the total deposited energy. The value for the simulated discriminator, applied at the estimated deposited energy at pixel level using the MPM, was adjusted independently for each detector layer. Figure 3(c) and figure 3(d) show the comparison of the spectrum of the singles between the experimental and simulated data for different threshold values in each detector layer. The local threshold value was set in each detector layer as high as possible avoiding the rejection of the events that were experimentally accepted which results in a value of $3 \mathrm{keV}$ in the first and $1 \mathrm{keV}$ in the second layer to the estimated deposited energy at pixel level. 
3.1.2. Energy spectra of coincidences The comparison of the energy spectra of the coincidences between experimental and simulated data is shown in figure 4 . From left to right, the deposited energy in the first detector layer, in the second detector layer and the summed deposited energy are shown. The shaded area around the experimental data values corresponds to 3-sigma interval considering Poisson distribution.

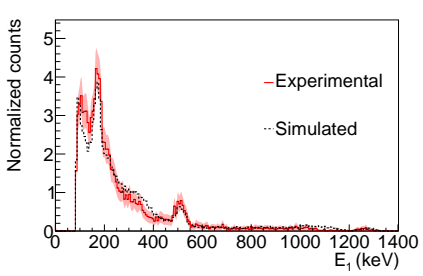

(a) first layer

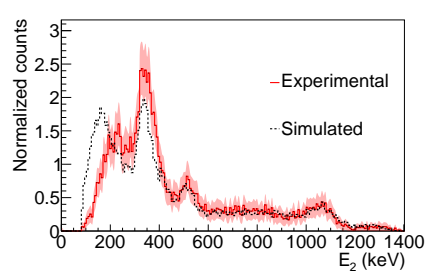

(b) second layer

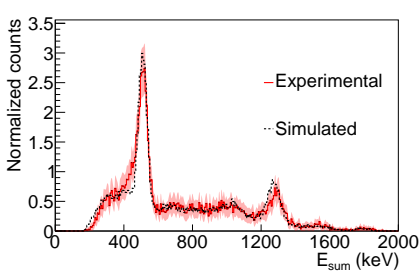

(c) sum

Figure 4: Simulated and experimental energy spectra of the coincidences.

3.1.3. Scatter Angles Figure 5 shows the comparison between the scattering angle obtained from geometrical method $\left(\theta_{G}\right.$, in X-axis) and the one obtained from Compton kinematics $\left(\theta_{C}\right.$, in $\mathrm{Y}$-axis) for $(5(\mathrm{a}))$ experimental data, $(5(\mathrm{~b}))$ simulated data assuming ideal energy and spatial resolution and (5(c)) applying the experimental energy and spatial resolution. In addition, the result obtained with the second version of the prototype (Barrio et al. 2018) composed of higher performance photo-detectors with a significantly better energy resolution of $5.9 \%$ FWHM at $511 \mathrm{keV}$ is shown in figure $5(\mathrm{~d})$.

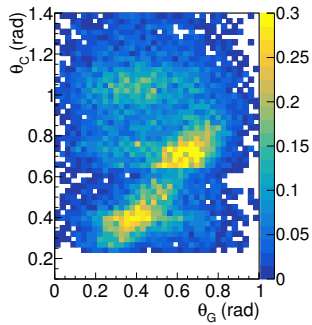

(a)

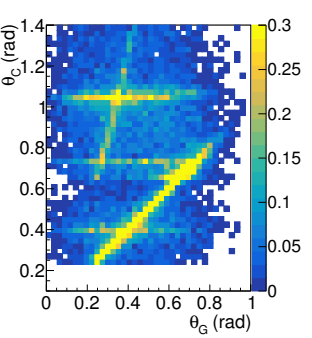

(b)

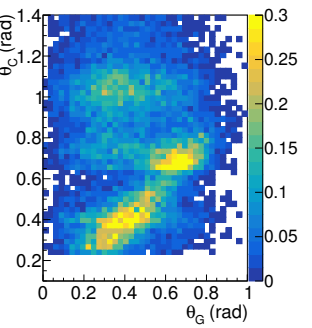

(c)

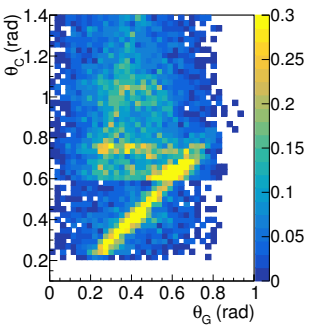

(d)

Figure 5: $\theta_{C}$ (Compton kinematics angle) vs $\theta_{G}$ (geometrical method) for (a) experimental data, (b) simulated data assuming ideal energy and spatial resolutions,

(c) applying experimental energy and spatial resolution and (d) experimental data taken with the second version of MACACO prototype.

Regarding the scatter angle, data can be classified into sequence coincidences that give rise to the diagonal line $\left(\theta_{G}=\theta_{C}\right)$ and the rest which are found outside the diagonal. The former consists of true sequence coincidences produced by one gammaray whereas the latter encompasses coincidences that degrade the image quality such as incorrectly ordered coincidences (due to back-scattering), partial absorption and random coincidences. The percentages of these invalid coincidences are shown in 
table 1 where randoms were divided into coincidences generated by different photons coming from the same decay, noted Single Decay Random (SDR) and coming from two different radioactive decays, noted Two-Decay Random (TDR). Among invalid coincidences four features can be identified according to figure 5 , three clearly visible horizontal lines and a vertical curved line. The horizontal lines corresponding to $\theta_{C} \simeq 0.40 \mathrm{rad}$ and $\theta_{C} \simeq 1.05 \mathrm{rad}$ come from an incorrect order in the sequence coincidence when forward scattering is assumed, for $1275 \mathrm{keV}$ and $511 \mathrm{keV}$ incident photons respectively. While $\theta_{C} \simeq 0.75 \mathrm{rad}$ is caused by random coincidences with a full absorption of $511 \mathrm{keV}$ photon in the first layer. Finally, the vertical curved line has its origin in the underestimation of the initial energy of the gamma-ray when the total deposited energy is below $600 \mathrm{keV}$ and the incident gamma ray corresponds to the $1275 \mathrm{keV}$ photon. This feature is clearly visible when ideal resolution is applied in the simulation (see figure $5(\mathrm{~b})$ ). It is worth noting that in the experimental data taken with the second version of MACACO prototype shown in figure $5(\mathrm{~d})$, this feature is also visible.

Table 1: Percentages of the different types of coincidences. The results for true coincidences are also shown for each incident energy $\left(E_{0}\right)$.

\begin{tabular}{lccc}
\hline & $E_{0}=511 \mathrm{keV}$ & $E_{0}=1275 \mathrm{keV}$ & all \\
\hline Coincidences & $7842(39.1 \%)$ & $8517(42.4 \%)$ & 20079 \\
\hline Single Decay randoms (SDR) & - & - & $15.4 \%$ \\
Two Decay randoms (TDR) & - & - & $3.1 \%$ \\
Back-scattering & $13.9 \%$ & $4.6 \%$ & $18.5 \%$ \\
Forward-scattering (FSC) & $25.2 \%$ & $37.8 \%$ & $63.0 \%$ \\
\hline FSC with full absorption & $14.8 \%$ & $7.3 \%$ & $22.1 \%$ \\
FSC with partial absorption & $10.0 \%$ & $21.1 \%$ & $31.1 \%$ \\
FSC with incorrect $E_{0}$ estimation & $0.4 \%$ & $9.4 \%$ & $9.8 \%$ \\
\hline
\end{tabular}

3.1.4. ARM The error of the scattering angle was studied by means of ARM and compared between experimental and simulated data. The angular error distribution is shown in figure 6 for (a) $511 \mathrm{keV}$ and (b) $1275 \mathrm{keV}$ estimated incident energy and for (c) all the coincidences showing a good agreement between experimental and simulated data. For $511 \mathrm{keV}$ incident energy, a second peak towards negative values is shown which mainly corresponds to the events with incorrect order or an underestimation of the initial energy. The ARM was studied for $1275 \mathrm{keV}$ estimated incident energy. The obtained value for the experimental data was $(13.5 \pm 0.2)^{\circ}$ whereas for simulated data was $(13.4 \pm 0.2)^{\circ}$.

3.1.5. Relative detection efficiency The obtained relative detection efficiency value for $1275 \mathrm{keV}$ incident photons was about $2.6 \times 10^{-3}$ for simulated data, whereas the measured value in a similar configuration reported in (Muñoz et al. 2017) was about $1.9 \times 10^{-3}$. An acceptable agreement was obtained between simulated data and the experimental reference value acquired with a similar configuration, especially considering that the crude analytical model of the discriminator threshold was adjusted 


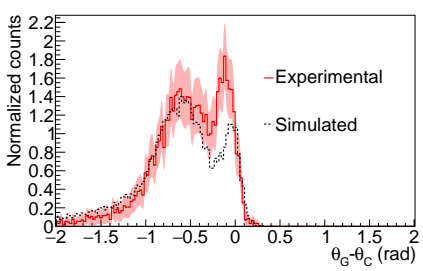

(a)

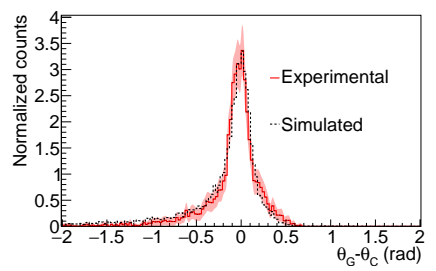

(b)

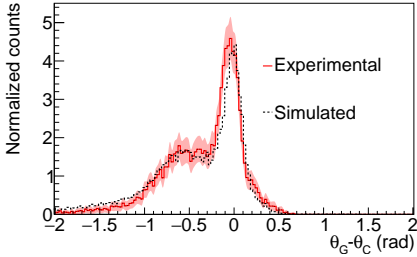

(c)

Figure 6: Angular error distribution for (a) $511 \mathrm{keV}$, (b) $1275 \mathrm{keV}$ estimated energy incident photon coincidences and (c) all the coincidences.

to accept more events than in the experimental scenario (see figure 3 ) which led to an overestimation of the detection efficiency of the system.

3.1.6. Back-projection onto the plane of the source Figure 7(a) and 7(b) show reconstructed images composed of $101 \times 101$ pixels of $1 \mathrm{~mm} \times 1 \mathrm{~mm}$ each from the back-projection onto the plane of the source of $1275 \mathrm{keV}$ estimated incident energy photons for experimental and simulated data respectively. A bias was presented in the reconstructed axial position for the experimental data where the source was reconstructed $\sim 12 \mathrm{~mm}$ closer to the first detector layer than for the simulated data.

The comparison of the normalized projections between both data sets along $x$ and $y$ orthogonal directions is shown respectively in figure $7(\mathrm{c})$ and $7(\mathrm{~d})$ when the simulated source is centered at $53 \mathrm{~mm}$ from the first layer. A displacement of about $4 \mathrm{~mm}$ in the $y$ direction is presented in the reconstructed experimental data (figure $7(\mathrm{~d})$ ) which is probably due to an error in the manual positioning of the source. The comparison of the normalized projections between both data sets when the source is located at the reconstructed experimental axial position, $41 \mathrm{~mm}$ from the first layer, along $x$ and $y$ orthogonal directions is shown respectively in figure $7(\mathrm{e})$ and $7(\mathrm{f})$ showing a better agreement.

\subsection{Towards more accurate results: simulation-based energy widow optimization}

Figure 8 shows the energy spectra of the coincidences assuming forward scattering generated by the different products of the decay of a ${ }^{22} \mathrm{Na}$ source of $847 \mathrm{kBq}$ activity: $511 \mathrm{keV}$ annihilation photons, $1275 \mathrm{keV}$ gamma-rays, SDR and TDR. The coincidences produced after a decay different than $\beta^{+}$(such as electron capture), which can be neglected, are also shown and labeled "others".

In order to minimize the SDR, the simulation allows to suggest an upper limit for the deposited energy in each layer and in the summed energy spectrum. In this study such limit corresponds to about $1200 \mathrm{keV}$ in each layer and to $1350 \mathrm{keV}$ in the summed energy spectrum. These acceptance windows reduce the SDR in a $21.4 \%$ and the TDR in a $16.0 \%$. In addition, the rejection of the coincidences with a deposited energy in the first layer comprised in $(490,550) \mathrm{keV}$ range will further reduce them in a $10.2 \%$, at the cost of rejecting $1.5 \%$ of good coincidences.

The angular error distribution of coincidences, excluding randoms (SDR, TDR), was studied for different low energy thresholds. Figure 9(a) and figure 9(b) show respectively the angular error distribution for impinging photons with an estimated 


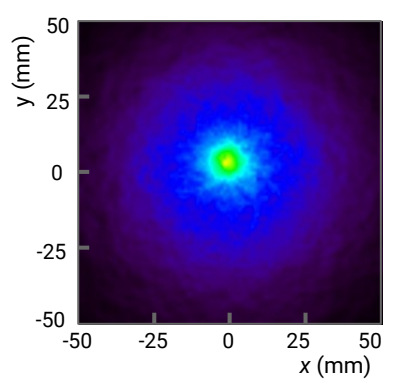

(a)

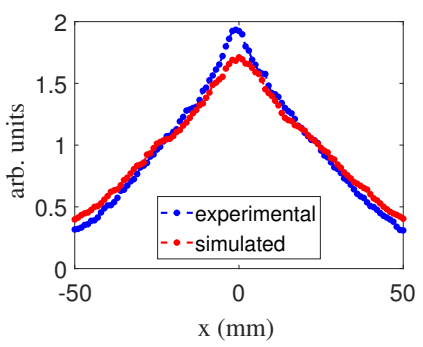

(c)

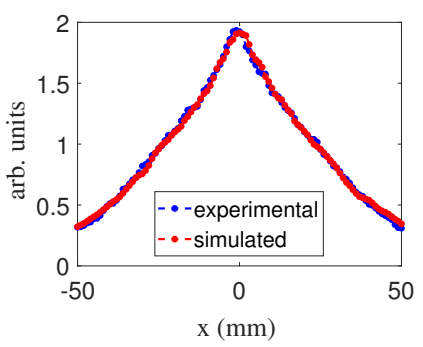

(e)

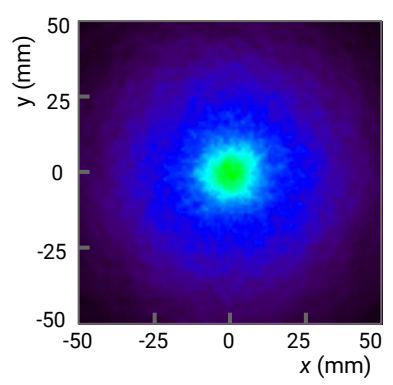

(b)

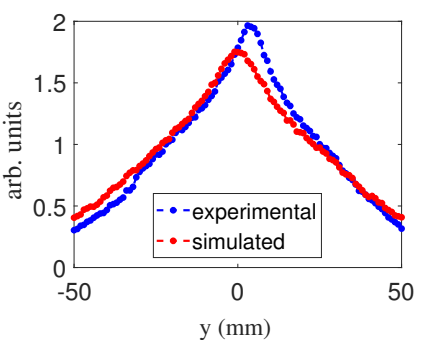

(d)

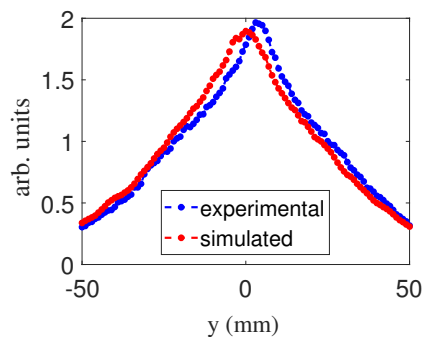

(f)

Figure 7: Back-projection images of $1275 \mathrm{keV}$ incident energy photons for (a) experimental and (b) simulated data. Comparison of the normalized projections along ((c),(e)) $x$ and ((d),(f)) y directions for the simulated source located at $53 \mathrm{~mm}$ from the first layer and located at the reconstructed axial position for experimental data (41 $\mathrm{mm}$ from the first layer) respectively.

energy of $511 \mathrm{keV}$ and $1275 \mathrm{keV}$ for different pairs of energy threshold values applied to the detector layers. It can be observed that when an energy threshold of $85 \mathrm{keV}$ was applied to both layers, which is in accordance with the reported value applied experimentally in (Muñoz et al. 2017), the number of coincidences for the incident energy of $511 \mathrm{keV}$ with small angular error ("small error" peak) was drastically reduced with respect to the distribution obtained without any energy threshold. For an incident energy of $1275 \mathrm{keV}$, the applied threshold rejected only about $10 \%$ of the coincidences. This result points out the need for low energy thresholds, especially in the first layer for relatively low gamma energies.

Regarding the angular error distribution, coincidences were classified into two 


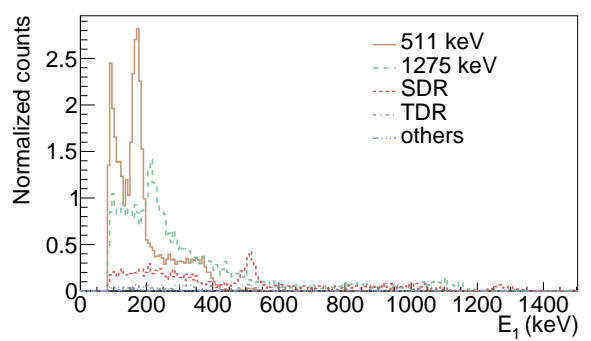

(a)

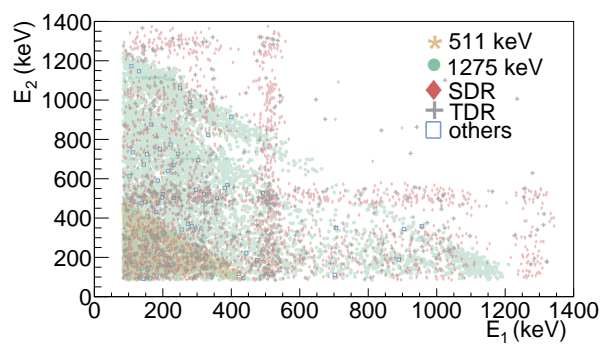

(c)

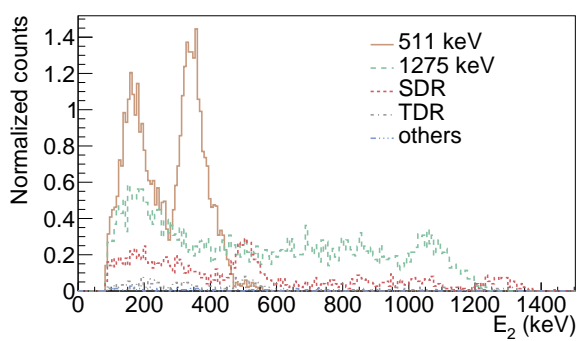

(b)

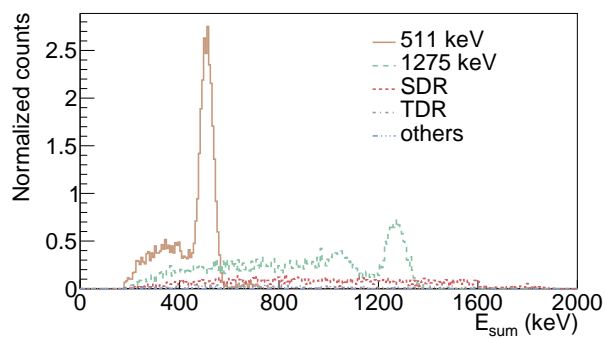

(d)

Figure 8: Deposited energies (a) in the first layer $\left(E_{1}\right),(\mathrm{b})$ in the second layer $\left(E_{2}\right)$, (c) 2D diagram $E_{2}$ vs $E_{1}$ and (d) summed energy spectrum of the coincidences.

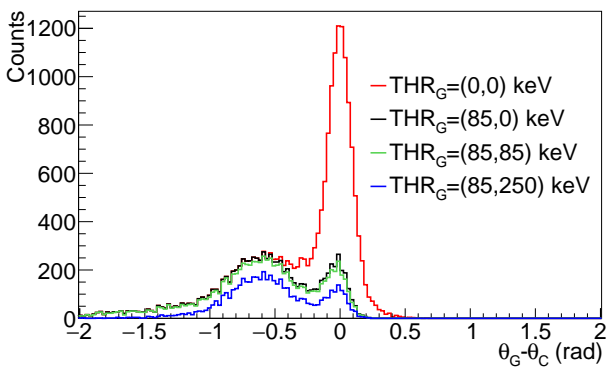

(a)

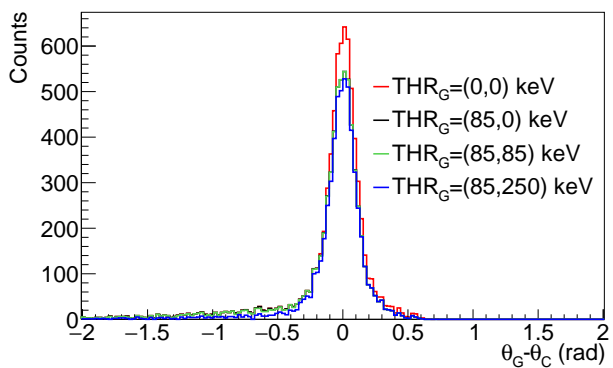

(b)

Figure 9: Angular error distribution for (a) $511 \mathrm{keV}$ and (b) $1275 \mathrm{keV}$ estimated incident energy. $\operatorname{THR}_{G}$ pair of values represents the energy threshold applied to the deposited energy in the first and second layer respectively.

groups delimited by $\sim 3 \sigma$, where $\sigma$ is the standard error of "small error" peaks. Coincidences with a large angular error value $(\geq 3 \sigma)$, considered as invalid coincidences in this study, represented the $\sim 33 \%$ of the total coincidences. The energy signature of these two data sets was characterized through the comparison between the deposited energy in the absorber $\left(E_{2}\right)$ and the deposited energy in the scatterer $\left(E_{1}\right)$, see figure 10. Two empty regions can be identified in the "small error" peak diagram (figure 10(a)) associated with the two incident energies of the photons, $511 \mathrm{keV}$ and $1275 \mathrm{keV}$. It turns out that the lower limits of the $E_{1}$ for these regions correspond approximately to the energies of recoil electrons associated with scattered photons 


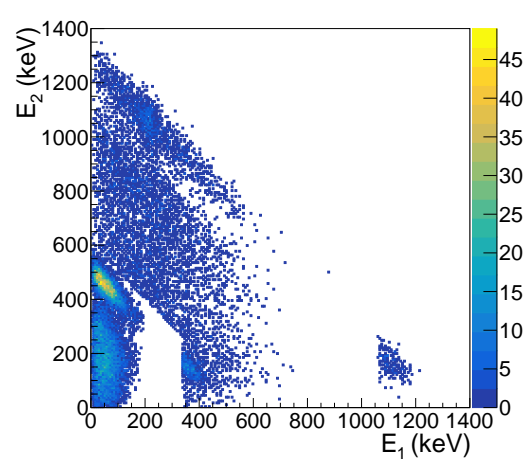

(a)

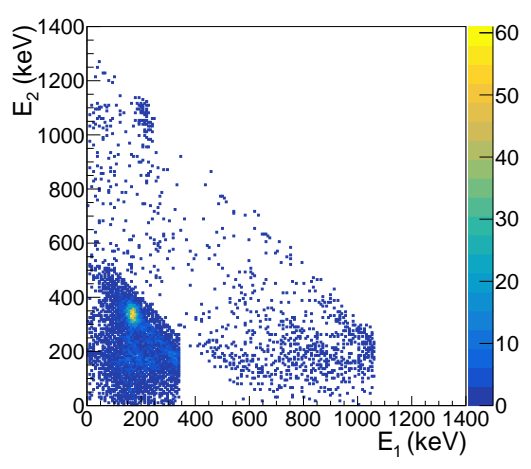

(b)

Figure 10: 2D diagram $E_{2}$ versus $E_{1}$ for coincidences with (a) small angular error and (b) large angular error delimited by three times the value of the standard error of the distribution.

that can not be detected due to the limited angular coverage of the absorber. For each incident energy, above this $E_{1}$ upper limit only back-scattering coincidences or coincidences with multiple interactions in the scatterer layer can been found. In our configuration, an upper energy threshold of $150 \mathrm{keV}$ applied to the scatterer for the estimated incident energy of $511 \mathrm{keV}$ reduced the fraction of the studied invalid coincides in a $\sim 62 \%$. Whereas an upper threshold of $700 \mathrm{keV}$ for the estimated incident energy of $1275 \mathrm{keV}$ reduced the fraction of the studied invalid coincidences in a $\sim 6 \%$.

The capability of the CCMod to define ad-hoc filters based on observable values to reduce the fraction of invalid coincidences was illustrated for the studied prototype.

\subsection{Comparison of the performance of different prototypes}

The relative detection efficiency and the angular resolution were studied as a function of the energy for both CC systems, MACACO and CLaRyS.

3.3.1. Efficiency Figure 11 shows the relative detection efficiency for both systems for different detection energy thresholds. The results for the reported energy threshold of $85 \mathrm{keV}$ for MACACO and the described operational thresholds for CLaRyS (50 keV to the scatterer and $100 \mathrm{keV}$ to the absorber) are shown in filled markers. The results for a lower energy threshold of $10 \mathrm{keV}$ applied to each detector type are shown in empty markers.

Figure 11 shows that a higher energy threshold leads to a larger reduction of the efficiency at low energies. This effect is more significant for MACACO prototype due to the smaller scattering acceptance angle which reduces the maximum allowed deposited energy in the first layer (in accordance with figure 9). In this low energy region, the detection efficiency increases as the energy increases. If the applied threshold is low enough, a second energy region (700-1000 keV) can be seen with an opposite behavior due to the decreasing photon interaction probability. This region is more visible for CLaRyS prototype since it is composed of thinner scatterer layers of lower atomic number material. Finally, for high incident energies, the contribution of pair creation to the detected efficiency may be non-negligible. This contribution can be clearly 


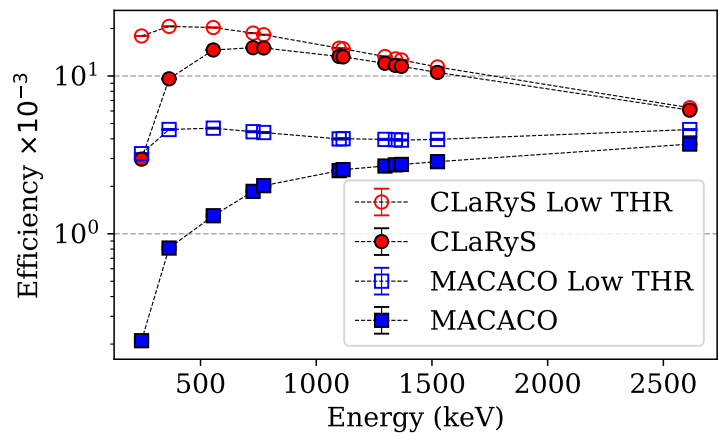

Figure 11: Relative detection efficiency as a function of the energy for CLaRyS (red circular markers) and MACACO (blue square markers) prototypes. The results for the low energy threshold are shown in empty markers.

observed for MACACO prototype when the low energy threshold was applied (blue empty square markers).

In general, the relative detection efficiency for CLaRyS prototype was always higher than for MACACO prototype mainly due to a three times thicker absorber and about two times higher scattering acceptance angle. It should also be noticed that MACACO does not have an optimized geometry, but just reproduces the experimental prototype layout and characterization results.

3.3.2. Angular resolution measure Figure 12 shows the ARM as a function of the incident energy for both prototypes. It monotonically decreases with increasing energy. The only exception was found for the highest energy considered due to the higher probability of pair creation events which reduces the signal to noise ratio. The ARM value for CLaRyS was always better than for MACACO prototype, with a reduction of about a factor $\sim 4$ for energies of $1 \mathrm{MeV}$.

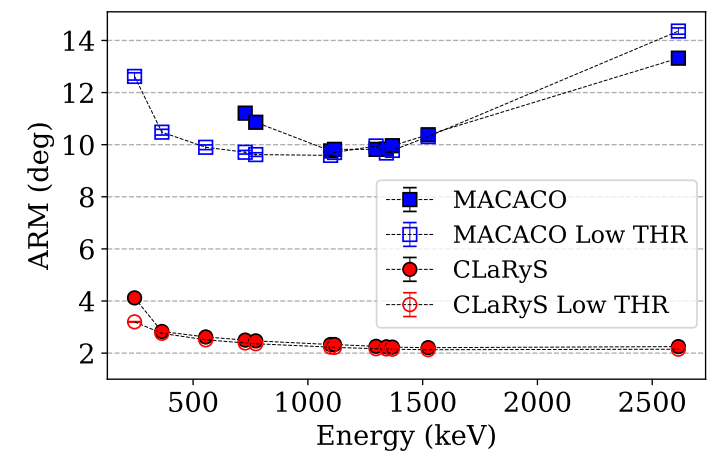

Figure 12: ARM as a function of the incident energy for CLaRyS (red circular markers) and MACACO (blue square markers) prototypes. The results for the low energy threshold are shown in empty markers. 


\section{Discussion and conclusions}

CCMod, a flexible alternative to simulate CCs, has been developed within GATE/Geant4 as a complement to the already existing PET and SPECT systems. We think that this generic module within GATE not only provides the tools for data analyses but will benefit from the broad range of medical experimental settings that can be conveniently simulated using macro language. CCMod has been designed to employ the same data structures, i.e. hits, singles and coincidences, as PET and SPECT systems. Thereby, the digitization modules may be applied interchangeably to all imaging devices. Moreover, additional modules can be added by users to take into account unforeseen situations. All the processing can be performed either on-the-fly or offline by the post-processing of simulation output files.

The predictions of CCMod were compared with experimental data in terms of energy spectra, angular resolution, efficiency and back-projection image reconstruction. The spectra of singles were used to assess the impact of the passive material and to adjust the discriminator threshold, at pixel level, in each detector independently. We observed that the passive material of the prototype (boards, holders...) played an important role in the scattering, resulting in a non-negligible contribution at low energies in both layers. The main parts were simulated improving the match with the experimental data. However, there was an underestimation of the passive material surrounding the sensitive detectors that was reflected in the simulated spectra of the singles. On the other hand, the employed analytical model to reproduce the experimental discriminator threshold at pixel level accepted slightly more events, mainly at low energies, than the one employed in the experimental data. This mismatch was more significant in the second layer due to its geometrical characteristics (thicker detector with smaller Aspect Ratio, ratio of length of the side of the detector to thickness). A good agreement between both simulated and experimental data regarding the spectra of the coincidences and the ARM was obtained despite small differences at low energies. The detection efficiency was compared to the measured value reported in (Muñoz et al. 2017) in a similar configuration. The obtained detection efficiency was higher by a factor $\sim 1.4$ than the experimental one which can be considered in good agreement due to the unknown experimental discriminator threshold that was rather underestimated to avoid the rejection of events registered experimentally. Regarding the back-projection image reconstruction, a good agreement was obtained between both data sets regardless of the small differences related to the error in the manual positioning of the source in the experimental data. In addition, the developed simulation was employed to identify undesired coincidences and reduce them by inferring filtering criteria, based on deposited energy, that can be applied to the experimental measurements with the prototype.

CCMod was employed to predict the response of the prototype under development by the CLaRyS collaboration and compare it with the performance of MACACO experimental prototype regarding the relative detection efficiency and the ARM. In general a higher efficiency was obtained for CLaRyS prototype than for MACACO prototype mainly due to a three times thicker absorber and about two times higher scattering acceptance angle. Regarding the ARM, the performance was about four times better for CLaRyS prototype with and angular resolution of about 2.3 degree at $1 \mathrm{MeV}$, mainly due to a better energy resolution in the scatterer. The main objective of this study was to show the ability of the developed module to simulate 
and reproduce the results of different existing prototypes, not to draw conclusions about their performances. It is worth noting that the two employed prototypes are in different development stages. MACACO is an experimental prototype driven by existing detector size and available funding resources at the time of construction, which is being improved, whereas CLaRyS is a prototype under construction which has not been assembled yet for acquisitions in coincidence between the two types of detectors.

In summary, a dedicated GATE Compton camera module has been developed which has been satisfactorily compared with experimental data and employed to study the performance of different prototypes. This extension of GATE will allow to easily compare under the same analyses the performance of different CC systems in medical experimental setting such as hadron therapy monitoring or nuclear medicine using CTbased patient phantoms. This module, CCMod, will be available in the next GATE release.

\section{Acknowledgments}

This work was performed within the framework of the SIRIC LYriCAN Grant INCaINSERM-DGOS-12563, the program "Investissements d'Avenir" (ANR-11-IDEX0007) operated by the ANR, the Ministerio de Ciencia, Innovación y Universidades (FPA2017-85611-R) and IFIC Severo Ochoa Excellence centre (SEV-2014-0398). This Project has received funding from the Labex PRIMES (ANR-11-LABX-0063) of Université de Lyon and PIC 2018 programme under the reference CSIC № 2018FR0032 and CNRS PICS08272.

\section{References}

Allison J, Amako K, Apostolakis J, Arce P, Asai M, Aso T, Bagli E, Bagulya A, Banerjee S, Barrand G, Beck B, Bogdanov A, Brandt D, Brown J, Burkhardt H, Canal P, Ott D, Chauvie S, Cho K and Yoshida H 2016 'Recent developments in Geant4' Nuclear Instruments and Methods in Physics Research Section A: Accelerators, Spectrometers, Detectors and Associated Equipment 835, 186-225.

Alnaaimi M, Royle G, Ghoggali W, Banoqitah E, Cullum I and Speller R 2011 'Performance evaluation of a pixellated Ge Compton camera' Physics in Medicine $\&$ Biology 56(12), 3473.

Andreyev A, Celler A, Ozsahin I and Sitek A 2016 'Resolution recovery for Compton camera using origin ensemble algorithm' Medical physics 43(8Part1), 4866-4876.

Arce P, Rato P, Canadas M and Lagares J I 2008 'GAMOS: A Geant4-based easy and flexible framework for nuclear medicine applications' in 'Nuclear Science Symposium Conference Record, 2008. NSS'08. IEEE' IEEE pp. 3162-3168.

Barrio J, Etxebeste A, Granado L, Muñoz E, Oliver J, Ros A, Roser J, Solaz C and Llosá G 2018 'Performance improvement tests of MACACO: A Compton telescope based on continuous crystals and SiPMs' Nuclear Instruments and Methods in Physics Research Section A: Accelerators, Spectrometers, Detectors and Associated Equipment 912, 48-52.

Bonifacio D A, Belcari N, Moehrs S, Moralles M, Rosso V, Vecchio S and Del Guerra A 2010 'A time efficient optical model for GATE simulation of a LYSO scintillation matrix used in PET applications' IEEE Transactions on Nuclear Science 57(5), 2483-2489.

Brown J M C, Dimmock M R, Gillam J E and Paganin D 2014 'A low energy bound atomic electron Compton scattering model for Geant4' Nuclear Instruments and Methods in Physics Research Section B: Beam Interactions with Materials and Atoms 338, 77-88.

Cabello J, Etxebeste A, Llosá G and Ziegler S I 2015 'Simulation study of PET detector limitations using continuous crystals' Physics in Medicine 83 Biology 60(9), 3673.

Calderón Y, Chmeissani M, Kolstein M and De Lorenzo G 2014 'Evaluation of Compton gamma camera prototype based on pixelated CdTe detectors' Journal of Instrumentation $\mathbf{9}(06), \mathrm{C} 06003$. 
Fontana M, Dauvergne D, Letang J M, Ley J and Testa É 2017 'Compton camera study for high efficiency SPECT and benchmark with Anger system' Physics in Medicine $\mathcal{G}$ Biology 62(23), 8794-812.

Gillam J E and Rafecas M 2016 'Monte-Carlo simulations and image reconstruction for novel imaging scenarios in emission tomography' Nuclear Instruments and Methods in Physics Research Section A: Accelerators, Spectrometers, Detectors and Associated Equipment 809, 76-88.

Golnik C, Bemmerer D, Enghardt W, Fiedler F, Hueso-González F, Pausch G, Römer K, Rohling H, Schöne S, Wagner L and Kormoll T 2016 'Tests of a Compton imaging prototype in a monoenergetic $4.44 \mathrm{MeV}$ photon field - a benchmark setup for prompt gamma-ray imaging devices' Journal of Instrumentation 11(06), P06009-P06009.

Grevillot L, Bertrand D, Dessy F, Freud N and Sarrut D 2011 'A Monte Carlo pencil beam scanning model for proton treatment plan simulation using GATE/Geant4.' Physics in Medicine \& Biology 56(16), 5203-5219.

Grevillot L, Bertrand D, Dessy F, Freud N and Sarrut D 2012 'GATE as a Geant4-based Monte Carlo platform for the evaluation of proton pencil beam scanning treatment plans.' Physics in Medicine 83 Biology 57(13), 4223-4244.

Gueth P, Dauvergne D, Freud N, Létang J M, Ray C, Testa E and Sarrut D 2013 'Machine learningbased patient specific prompt-gamma dose monitoring in proton therapy.' Physics in Medicine G Biology 58(13), 4563-4577.

Han L, Rogers W L, Huh S S and Clinthorne N 2008 'Statistical performance evaluation and comparison of a Compton medical imaging system and a collimated Anger camera for higher energy photon imaging' Physics in Medicine 83 Biology 53(24), 7029.

Harkness L, Arce P, Judson D, Boston A, Boston H, Cresswell J, Dormand J, Jones M, Nolan P, Sampson J et al. 2012 'A Compton camera application for the GAMOS Geant4-based framework' Nuclear Instruments and Methods in Physics Research Section A: Accelerators, Spectrometers, Detectors and Associated Equipment 671, 29-39.

Harkness L, Boston A, Boston H, Cooper R, Cresswell J, Grint A, Nolan P, Oxley D, Scraggs D, Beveridge $\mathrm{T}$ et al. 2009 'Optimisation of a dual head semiconductor Compton camera using Geant4' Nuclear Instruments and Methods in Physics Research Section A: Accelerators, Spectrometers, Detectors and Associated Equipment 604(1-2), 351-354.

Hilaire E, Sarrut D, Peyrin F and Maxim V 2016 'Proton therapy monitoring by Compton imaging: influence of the large energy spectrum of the prompt- $\gamma$ radiation' Physics in Medicine \& Biology 61(8), 3127.

Huisman B F B, Letang J M, Testa E and Sarrut D 2016 'Accelerated prompt gamma estimation for clinical proton therapy simulations.' Physics in medicine 85 biology 61, 7725-7743.

Jan S, Benoit D, Becheva E, Carlier T, Cassol F, Descourt P, Frisson T, Grevillot L, Guigues L, Maigne L, Morel C, Perrot Y, Rehfeld N, Sarrut D, Schaart D R, Stute S, Pietrzyk U, Visvikis D, Zahra N and Buvat I 2011 'GATE V6: a major enhancement of the GATE simulation platform enabling modelling of CT and radiotherapy.' Physics in Medicine $\mathscr{G}$ Biology 56(4), 881-901.

Jan S, Santin G, Strul D, Staelens S, Assie K, Autret D, Avner S, Barbier R, Bardies M, Bloomfield $\mathrm{P}$ et al. 2004 'Gate: a simulation toolkit for PET and SPECT' Physics in Medicine 83 Biology 49(19), 4543.

Karimian A, Jia B, Raisali G and Rahimi F 2009 'Design of a new Compton camera by Monte Carlo method and assessment its important parameters affecting image quality' in 'World Congress on Medical Physics and Biomedical Engineering, September 7-12, 2009, Munich, Germany' Springer pp. 414-416.

Kishimoto A, Kataoka J, Nishiyama T, Taya T and Kabuki S 2015 'Demonstration of threedimensional imaging based on handheld Compton camera' Journal of Instrumentation 10(11), P11001-P11001.

Kormoll T, Fiedler F, Schöne S, Wüstemann J, Zuber K and Enghardt W 2011 'A Compton imager for in-vivo dosimetry of proton beams - a design study' Nuclear Instruments and Methods in Physics Research Section A: Accelerators, Spectrometers, Detectors and Associated Equipment 626, 114-119.

Kurosawa S, Kubo H, Ueno K, Kabuki S, Iwaki S, Takahashi M, Taniue K, Higashi N, Miuchi K, Tanimori T, Kim D and Kim J 2012 'Prompt gamma detection for range verification in proton therapy' Current Applied Physics 12(2), 364-368.

Lee H R, Kim S H, Park J H, Jung W G, Lim H and Kim C H 2017 'Prototype system for proton beam range measurement based on gamma electron vertex imaging' Nuclear Instruments and Methods in Physics Research Section A: Accelerators, Spectrometers, Detectors and Associated Equipment 857, 82-97.

Lee S H, Seo H, Park J H, Park S H, Lee J S, Lee J H, Lee C S and Kim C H 2009 'CIS: a GUI- 
based software system for Monte Carlo simulation of Compton camera' Nuclear Technology 168(1), 55-60.

Lehner C E, He Z and Zhang F 2004 ' $4 \pi$ Compton imaging using a 3-D position-sensitive CdZnTe detector via weighted list-mode maximum likelihood' IEEE Transactions on Nuclear Science 51(4), 1618-1624.

Llosá G, Bernabeu J, Burdette D, Chesi E, Cindro V, Clinthorne N, Honscheid K, Kagan H, Lacasta C, Mikuž M, Modesto P, Rogers W, Steinberg J, Studen A, Weilhammer P, Zhang $\mathrm{L}$ and Žontar D 2006 'Results of a first demonstrator prototype of a Compton prostate probe' Nuclear Instruments and Methods in Physics Research Section A: Accelerators, Spectrometers, Detectors and Associated Equipment 569(2), 277-280.

Maier D, Blondel C, Delisle C, Limousin O, Martignac J, Meuris A, Visticot F, Daniel G, Bausson P A, Gevin O et al. 2017 'Second generation of portable gamma camera based on Caliste CdTe hybrid technology' Nuclear Instruments and Methods in Physics Research Section A: Accelerators, Spectrometers, Detectors and Associated Equipment.

McCleskey M, Kaye W, Mackin D, Beddar S, He Z and Polf J 2015 'Evaluation of a multistage CdZnTe Compton camera for prompt gamma imaging for proton therapy' Nuclear Instruments and Methods in Physics Research Section A: Accelerators, Spectrometers, Detectors and Associated Equipment 785, 163-169.

Mihailescu L, Vetter K, Burks M, Hull E and Craig W 2007 'SPEIR: a Ge Compton camera' Nuclear Instruments and Methods in Physics Research Section A: Accelerators, Spectrometers, Detectors and Associated Equipment 570(1), 89-100.

Mizumoto T, Tomono D, Takada A, Tanimori T, Komura S, Kubo H, Matsuoka Y, Mizumura Y, Nakamura K, Nakamura S et al. 2015 'A performance study of an electron-tracking Compton camera with a compact system for environmental gamma-ray observation' Journal of Instrumentation 10(06), C06003.

Montémont G, Bohuslav P, Dubosq J, Feret B, Monnet O, Oehling O, Skala L, Stanchina S, Verger L and Werthmann G 2017 'NuVISION: a portable multimode gamma camera based on HiSPECT imaging module' in '2017 IEEE Nuclear Science Symposium and Medical Imaging Conference (NSS/MIC)' IEEE pp. 1-3.

Muñoz E, Barrio J, Etxebeste A, Ortega P G, Lacasta C, Oliver J F, Solaz C and Llosá G 2017 'Performance evaluation of MACACO: a multilayer Compton camera' Physics in Medicine $\mathcal{G}^{3}$ Biology 62(18), 7321-41.

Novikova E, Stickman M, Gwon C and B.F.Phlips 2006 'Designing sword-software for optimization of radiation detectors' in 'Nuclear Science Symposium Conference Record, 2006. IEEE' IEEE pp. 607-61.

Odaka H, Ichinohe Y, Takeda S, Fukuyama T, Hagino K, Saito S, Sato T, Sato G, Watanabe S, Kokubun M et al. 2012 'High-resolution Compton cameras based on Si/CdTe doublesided strip detectors' Nuclear Instruments and Methods in Physics Research Section A: Accelerators, Spectrometers, Detectors and Associated Equipment 695, 179-183.

Ordonez C E, Bolozdynya A and Chang W 1997 'Doppler broadening of energy spectra in Compton cameras' in 'Nuclear Science Symposium, 1997. IEEE' Vol. 2 IEEE pp. 1361-1365.

Peterson S, Robertson D and Polf J 2010 'Optimizing a three-stage Compton camera for measuring prompt gamma rays emitted during proton radiotherapy' Physics in Medicine 85 Biology $\mathbf{5 5}(22), 6841$.

Polf J C, Avery S, Mackin D S and Beddar S 2015 'Imaging of prompt gamma rays emitted during delivery of clinical proton beams with a Compton camera: feasibility studies for range verification' Physics in Medicine \& Biology 60(18), 7085.

Richard M H, Chevallier M, Dauvergne D, Freud N, Henriquet P, Foulher F L, Letang J M, Montarou G, Ray C, Roellinghoff F, Testa E, Testa M and Walenta A H 2011 'Design guidelines for a double scattering Compton camera for prompt-gamma imaging during ion beam therapy: A Monte Carlo simulation study' IEEE Transactions on Nuclear Science 58(1), 87-94.

Rohling H, Priegnitz M, Schoene S, Schumann A, Enghardt W, Hueso-González F, Pausch G and Fiedler F 2017 'Requirements for a Compton camera for in vivo range verification of proton therapy' Physics in Medicine 83 Biology 62(7), 2795.

Sarrut D, Bardiès M, Boussion N, Freud N, Jan S, Létang J M, Loudos G, Maigne L, Marcatili S, Mauxion T et al. 2014 'A review of the use and potential of the gate Monte Carlo simulation code for radiation therapy and dosimetry applications' Medical physics 41(6Part1).

Seo H, Kim C H, Park J H, Kim J K, Lee J H, Lee C S and Lee J S 2010 'Development of double-scattering-type Compton camera with double-sided silicon strip detectors and $\mathrm{NaI}(\mathrm{tl})$ scintillation detector' Nuclear Instruments and Methods in Physics Research Section A: Accelerators, Spectrometers, Detectors and Associated Equipment 615(3), 333-339. 
Seo H, Lee S H, Kim C H, An S H, Lee J H and Lee C S 2008 'Optimal geometrical configuration of a double-scattering Compton camera for maximum imaging resolution and sensitivity' Nuclear Instruments and Methods in Physics Research Section A: Accelerators, Spectrometers, Detectors and Associated Equipment 591(1), 80-83.

Seo H, Park J H, Ushakov A, Kim C H, Kim J K, Lee J H, Lee C S and Lee J S 2011 'Experimental performance of double-scattering Compton camera with anthropomorphic phantom' Journal of Instrumentation 6(01), C01024.

Sinclair L, Hanna D, MacLeod A and Saull P 2009 'Simulations of a scintillator Compton gamma imager for safety and security' IEEE Transactions on Nuclear Science 56(3), 1262-1268.

Solevi P, Muñoz E, Solaz C, Trovato M, Dendooven P, Gillam J E, Lacasta C, Oliver J F, Rafecas M, Torres-Espallardo I et al. 2016 'Performance of MACACO Compton telescope for ionbeam therapy monitoring: first test with proton beams' Physics in Medicine 8$\}$ Biology 61(14), 5149.

Strydhorst J and Buvat I 2016 'Redesign of the GATE PET coincidence sorter' Physics in Medicine Es Biology 61(18), N522-N531.

Takeda S, Odaka H, nosuke Ishikawa S, Watanabe S, Aono H, Takahashi T, Kanayama Y, Hiromura $\mathrm{M}$ and Enomoto S 2012 'Demonstration of in-vivo multi-probe tracker based on a Si/CdTe semiconductor Compton camera' IEEE Transactions on Nuclear Science 59(1), 70-76.

Thirolf P, Aldawood S, Böhmer M, Bortfeldt J, Castelhano I, Dedes G, Fiedler F, Gernhäuser R, Golnik C, Helmbrecht S, Hueso-González F, v.d. Kolff H, Kormoll T, Lang C, Liprandi S, Lutter R, Marinšek T, Maier L, Pausch G, Petzoldt J, Römer K, Schaart D and Parodi K 2016 'A Compton camera prototype for prompt gamma medical imaging' EPJ Web of Conferences 117, 05005 .

Todd R, Nightingale J and Everett D 1974 'A proposed $\gamma$ camera' Nature 251(5471), 132.

Zoglauer A, Andritschke R, Boggs S E, Schopper F, Weidenspointner G and Wunderer C B 2008 'MEGAlib: simulation and data analysis for low-to-medium-energy gamma-ray telescopes' in 'Space Telescopes and Instrumentation 2008: Ultraviolet to Gamma Ray' Vol. 7011 International Society for Optics and Photonics p. 70113F.

Zoglauer A, Andritschke R and Schopper F 2006 'MEGAlib-the medium energy gamma-ray astronomy library' New Astronomy Reviews 50(7-8), 629-632.

Zoglauer A and Kanbach G 2003 'Doppler broadening as a lower limit to the angular resolution of next-generation Compton telescopes' in 'X-Ray and Gamma-Ray Telescopes and Instruments for Astronomy' Vol. 4851 International Society for Optics and Photonics pp. 1302-1310. 